\title{
Inclinación a la infidelidad y modos de amar en estudiantes universitarios
}

\author{
Inclination to infidelity and ways of loving in university students
}

\author{
Carla Maité Condori Yanapa', Fernando Pari Belizarioº
}

\begin{abstract}
RESUMEN
Objetivo: determinar la relación entre la inclinación a la infidelidad y los modos de amar en estudiantes universitarios. Metodología: de diseño no experimental, enfoque cuantitativo, tipo correlacional, corte transversal. La población estuvo conformada por 195 estudiantes universitarios, con edades oscilantes entre 17 a 36 años, el muestreo fue no probabilístico por conveniencia. Resultados: se encontró correlación significativa entre inclinación a la infidelidad y los estilos de amor ludus y manía $(p<0.05)$, sin embargo, no se encontró relación significativa con los estilos de amor: Eros, storge, pragma y ágape $(p>0.05)$. Conclusión: sí existe relación significativa entre tendencia a la infidelidad y los estilos de amar, ludus y manía, ya que las conductas de estos tipos de amor tienden al amor efímero, inseguro, con poco compromiso.
\end{abstract}

Palabras clave: Estilos de amar, infidelidad, ludus, manía, eros.

\section{ABSTRACT}

Objective: to determine the relationship between the inclination to infidelity and the ways of loving in university students. Methodology: non-experimental design, quantitative approach, correlational type, cross-sectional. The population consisted of 195 university students, with ages ranging from 17 to 36 years, the sampling was non-probabilistic for convenience. Results: a significant correlation was found between the inclination to infidelity and the ludus and mania love styles $(p<0.05)$, however, no significant relationship was found with the love styles: Eros, storge, pragma and agape $(p>0.05)$. Conclusion: there is a significant relationship between the tendency to infidelity and the styles of love, ludus and mania, since the behaviors of these types of love tend to ephemeral, insecure love, with little commitment.

Keywords: Styles of loving, infidelity, ludus, mania, eros.

${ }^{1}$ Universidad Peruana Unión, Juliaca, Perú. 


\section{INTRODUCCIÓN}

El Comercio reporta que en el Perú el $71 \%$ de hombres reconoce haber engañado a su pareja, mientras que en las mujeres es el $50 \%$, mediante estos datos se puede observar que pocos años atrás, la infidelidad era más prominente en varones que en mujeres, en cambio en la actualidad es igual tanto en mujeres como en varones (Comercio, 2010).

Así también, Quevedo (1997) realizó una investigación en Perú, titulada: "Actitudes hacia la infidelidad conyugal por sexo, edad y tiempo de convivencia en parejas en cuatro distritos de Lima metropolitana", dónde se halló que $64 \%$ de la muestra estima que en el hombre es común que mantengan relaciones sexuales fuera del matrimonio, en la cual las mujeres no consideran a la insatisfacción sexual como motivo o excusa de infidelidad. Por otra parte, el $70 \%$ se mostró disconforme con restringir la diversidad de su comportamiento sexual en el matrimonio, admitiendo las necesidades sexuales como factor valioso para la infidelidad conyugal.

Del mismo modo en el Hospital Distrital de Laredo, ubicado en Perú se realizó un estudio con pacientes entre 20 y 40 años de edad, en el cual se evidenció que la infidelidad sexual ocurre constantemente con un $68 \%$, dónde resaltan más los varones, ya que la diferencia estadística significativa entre mujeres y varones es $(p<0.01)$ y en satisfacción sexual e infidelidad es $(p<0.01)$ (Chávez y Juárez, 2016).

Por otro lado, Vargas (2011) en la investigación que realizó en Colombia; cuyo título es: "Estudio comparativo en torno a la infidelidad, sexo casual, uso de lencería, ataduras e inmovilizaciones, piropos, sexo oral, orgías y encuentros con 16 prostitutas en hombres y mujeres en los sectores populares y medio-altos en Bogotá, Colombia", donde llegó a la conclusión de que el $91 \%$ de los varones abandonarían a su pareja si esta no los complace sexualmente, mientras que en el caso de las mujeres sólo el 31\% lo harían.

Según Lewandowski y Ackerman (2006), refieren que más del $50 \%$ de las personas casadas en sociedades occidentales están implicadas con la infidelidad, en cambio desde un punto de vista evolutivo la persona puede mantenerse durante un tiempo de 2 a 3 años con una sola pareja, pero conforme va pasando el tiempo es usual que uno de los individuos tiende a buscar una nueva pareja.
Se evidencia que, a través de casos terapéuticos con problemas de infidelidad, las personas infieles priorizan compartir sus necesidades (sexuales y emocionales), preferencias, valores y anhelos con una tercera persona que, con su pareja, sumado que la infidelidad es un síntoma de la mala comunicación de pareja (Garciandia y Samper, 2012).

Es por ello que la presente investigación pretende estudiar la inclinación hacia la infidelidad y los modos de amar, siendo un fenómeno aparentemente común, puesto que la manera de amar de cada persona es diferente, ya sea con mayor o menor intensidad, influyendo en las conductas infieles, posiblemente por la percepción que se tenga del amor, donde se agrupan pensamientos distorsionados o arraigados. De esta manera se aporta información a los estudios de problemas de pareja, resaltando que la infidelidad causa un impacto e inestabilidad emocional tanto en la víctima, como en el infiel, desorganizando a la persona en su ámbito laboral, familiar, académico, social, etc, asimismo se evidencia la manera de amar que tienen las personas con conducta fiel e infiel.

Se define al amor como un grupo de pensamientos, sentimientos, acciones, comunicación verbal y no verbal (Yela, 2001). Asimismo, se le atribuye como una construcción cultural ya que en cada época se tiene una percepción diferente del amor (De Roda, Martínez-Íñigo, de Paúl, y Yela, 1999). La Real Academia Española da a entender que el amor es un sentimiento intenso del ser humano brindando afecto y entrega hacia alguien o algo, anhelando reciprocidad, dando la sensación de estar completos y alegres, también referido a la unión sexual (Real Academia Española, 2014).

Es posible que se tenga dificultades para medir el amor, sin embargo, puede ser cuantificado, ya que las personas al momento de percibirlo suelen medir el nivel de amor que sienten por sus similares, y las dificultades que surgen al medir la variable pueden ser similares al medir la depresión, personalidad e inteligencia (Cassepp-Borges y Pasquali, 2012).

Lee (1973) plantea 6 tipos de amor donde tres son principales: Eros - amor erótico: Un estilo de amor que se inclina a valorar más el atractivo físico de su pareja; ludus - amor lúdico: Toma al amor y sexo como un juego, no genera 
compromiso o vínculos emocionales; storgeamor amistoso: Este estilo de amor se centra en la amistad y afecto, ignorando el aspecto físico o satisfacción sexual. Tres secundarios: Pragma - amor pragmático: Se busca cualidades de compatibilidad con la otra persona, basándose en su estatus social, apariencia física, religión, nivel de educación, antecedentes familiares; manía amor obsesivo: Se presentan características inestables, obsesivas, celosas e inseguras con su pareja; ágape - amor altruista: Se caracteriza por ser un amor intenso, que entrega todo sin esperar nada a cambio.

Por otra parte, Vargas e Ibañez (2008) definen cómo infidelidad a la desobediencia del pacto de fidelidad, englobando el campo sexual y emocional, esta idea puede variar según las creencias establecidas sobre el amor. Esto se puede explicar por la descarga de químicos en el cerebro que causan placer y estados eufóricos, dichos estados van cambiando durante el periodo de relación de pareja (Fisher, 2004).

Además, son muchos los factores relacionados a la infidelidad, esta actúa como una causa principal de la violencia familiar o violencia hacia la mujer (Alva, 2017). También se puede observar la relación de la infidelidad y redes sociales, la cual se considera como influyente indirecto de la infidelidad, mediante éstas las personas se contactan fácilmente, ya sea con su expareja o con otros individuos, siendo proclives a la infidelidad ya estando en otra relación; demostrando que la adicción a redes sociales crea un impacto en las relaciones de pareja, creando falta de comunicación, desequilibrio emocional y baja autoestima (Cruz y Rodríguez, 2016).

Es decir, la infidelidad es un asunto común observado en las parejas, aun cuando las normas de la sociedad se oponen a ésta. Alva (2017) menciona que la infidelidad es quebrantar una relación, ocurriendo por diferentes causas (inestabilidad emocional, carencias afectivas falta de gratificación sexual); existe una clasificación extendida sobre tipos de infidelidad, como la espontánea, permanente, con o sin relaciones sexuales y la infidelidad por huir de la monotonía.

Del mismo modo, la infidelidad suele ser diferente en ambos sexos, provocando consecuencias en cada uno de distinta manera, por ejemplo en el sexo femenino suelen ser infieles por las búsqueda de emociones, afecto, amor, atención y salir de lo cotidiano, dándose a entender como un síntoma de la inestable relación sentimental; en cambio el sexo masculino no lo percibe como una crisis de la relación; por lo tanto, no siente que ser infiel sea un escape, exonerándose de culpabilidad por dicho acto (Malek, 2015).

Ahora bien, en Estados Unidos, se investigó tendencias de infidelidad mediante la aplicación móvil "Tinder", la cual permite conocer personas eligiendo la que más te agrade, la mayoría de usuarios buscaba encontrar una pareja sexual esporádica, indicando que la aplicación les incitaba o facilitaba a ser infiel, de este modo se pudo evidenciar que la sexualidad y la intención de ser infiel se asoció al uso de Tinder (Weiser et al., 2018). De la misma manera Alavi, Kye Mei, y Mehrinezhad (2018), indican que la infidelidad es un factor de riesgo para la salud mental de la pareja sentimental y puede ocasionarse por los diferentes tipos de personalidad, tal como los autores lo denominan la triada oscura, hallando que la infidelidad tiene relación significativa con el narcisismo, maquiavelismo y psicopatía.

Por otro lado, en España se investigó factores asociados a la relación sexual satisfactoria, donde ocho de cada diez españolas consideran que es muy importante tener una relación sexual satisfactoria, pues se percibe como un elemento desestresante que mejora su estado de ánimo, evidenciando pensamientos y personalidad sexual abierta, llegando a tener mayor número de parejas, siendo proclives a ser infieles puesto que lo ven como algo positivo. Asimismo, las personas son infieles porque buscan revalorar su rol de pareja, demostrando que existe alguien más que les puede ofrecer aquello que carecen en la relación (Puicón y Salas, 2016; Romero, Rivera y Loving, 2007).

Siguiendo con, Chu (2020) refiere que en Perú la infidelidad afecta a una persona a nivel del sistema cognitivo, emocional y conductual, ya que podrían presentarse episodios depresivos, síntomas ansiosos, crisis emocionales y una baja autoestima, porque es común que las personas víctimas de la infidelidad se menosprecien a sí mismas, considerando que tales afectaciones tienden a durar un mínimo de 6 meses; sin embargo, si estas perduran años llegan a convertirse en problemas psicológicos crónicos; y si existe una familia de por medio trae como consecuencia desorganización, afección a los hijos y destrucción de la confianza de pareja. 
También, en Perú se argumenta que la infidelidad se genera por las presiones sociales de una sociedad machista, donde varones se jactan de tener muchas parejas, debido a esto el entorno expuesto es provocado a ser infiel, es decir cuanto más parejas se tenga, más exitoso será el varón, no obstante se añade que la infidelidad también se genera por aquellas mujeres que desconocen su propio cuerpo, avergonzandose de sí mismas, limitándose en la intimidad, como consecuencia el hombre busca en otra pareja lo que no obtiene en la suya. También se menciona que las mujeres se muestran más reacias a aceptar que han sido infieles, dado que el porcentaje de infidelidad es igual en hombres y mujeres (Puicón y salas, 2016). Maldonado (2018), en su investigación encontró una relación negativa significativa entre sus variables tendencia a la infidelidad y actitudes hacia el machismo, dónde se concluye que si existe menos tendencia a la infidelidad entonces es menor la actitud machista, siendo su población de 323 varones.

En otro estudio Romero, Romero y Arellano (2017), refieren que las mujeres tienen tendencia a la infidelidad porque sufren de violencia psicológica y física, especialmente emocional, afectando el área familiar, social y laboral.

Con relación al apego, sí se encuentra un vínculo con la infidelidad, puesto que las personas con menor índice de conducta infiel evidencian un apego seguro, en cambio personas con apego preocupante tienen mayores probabilidades de tener creencias de infidelidad (Urrego, Gaitan y Umbarila, 2016). Asimismo, se encontró que el clima social familiar y la tendencia a la infidelidad no tienen correlación, ya que la población refería adecuado clima social familiar, y su tendencia a la infidelidad era medio, dando a entender que la tendencia a la infidelidad está vinculado al sistema de creencias que tiene la persona (Ramirez, 2017).

En un estudio ejecutado por Marzec y Lukasik (2017) en base a su muestra de su investigación, en Polonia, proponen que las personas con los estilos de amar eros, storge y/o pragma presentan orientación a estrategias socio sexuales restringidas, insinuando relaciones a largo plazo, mientras que el estilo de amar ludus se ve inclinada a las relaciones pasajeras.

Siguiendo con Pedrós (2016), llevó a cabo un estudio que tuvo como objetivo determinar la relación que existe entre las diferentes visiones sobre el concepto del amor y su relación con los límites de la infidelidad y las actitudes hacia la violencia de género, tomando en cuenta las dos primeras variables, obtuvo que, la infidelidad se relaciona con el estilo de amar ludus, pragma y manía.

En otra investigación sobre formas de amar e infidelidad en estudiantes universitarios, se encontró que la infidelidad tiene una relación significativa con los estilos de amar lúdico, obsesivo y erótico (Delgado, 2020). De igual manera, Urizar (2018) encontró que la infidelidad tiene relación con los estilos de amar, destacando el estilo de amar eros.

Por otro lado, Couch, Baughman y Derow (2017) determinaron que cuando una relación sentimental finaliza, aquellas personas que evidenciaron un estilo de amar maníaco durante el tiempo de relación, más adelante presentan respuestas psicológicas como depresión, ansiedad o trauma.

El objetivo general es determinar si existe relación entre inclinación a la infidelidad y los modos de amar; teniendo como objetivos específicos: Determinar si existe relación entre inclinación a la infidelidad y el modo de amar eros, determinar si existe relación entre inclinación a la infidelidad y el modo de amar ludus, determinar si existe relación entre inclinación a la infidelidad y el modo de amar storge, determinar si existe relación entre inclinación a la infidelidad y el modo de amar pragmático, determinar si existe relación entre inclinación a la infidelidad y el modo de amar maníaco, determinar si existe relación entre inclinación a la infidelidad y el estilo de amar ágape.

\section{METODOLOGÍA}

El tipo de investigación es correlacional, de diseño no experimental, corte transversal y enfoque cuantitativo. Correlacional porque se pretende determinar si existe relación entre la variable tendencia a la infidelidad y modos de amar; de la misma manera de corte transversal porque la recolección de datos es por única vez en la línea del tiempo, asimismo, de diseño no experimental porque no se manipuló ninguna variable, finalmente, de enfoque cuantitativo por qué se hace uso de la estadística para probar 
las hipótesis planteadas (Hernández, Fernández y Baptista, 2014).

\section{Participantes}

El muestreo fue probabilístico por conveniencia, conformado por 195 estudiantes universitarios de diferentes carreras universitarias, la población fue la adecuada para este tipo de investigación; se excluyó a evaluados que nunca tuvieron enamorado(a) o pareja. En la tabla 1 se muestra el análisis sociodemográfico de la población, donde el $70.3 \%$ pertenece al género femenino, mientras que el $29.7 \%$ pertenece al género masculino. En cuanto a la edad hay un rango desde los 17 años hasta los 36 años, las edades más representativas son las de 21,20 y 18 años, la cantidad de enamorados más representativa son uno y dos, el $47.2 .5 \%$ está en una relación sentimental, la carrera profesional con más frecuencia es la de psicología con un $46.7 \%$, en religión el $44.6 \%$ es adventista, respecto a procedencia el número más representativo es de la sierra con un $73.8 \%$.

Tabla 1

Tabla de datos sociodemográficos de la población de estudio.

\begin{tabular}{llll} 
Variables sociodemográficas & & Frecuencia & Porcentaje \% \\
Edad & 17 a 36 años & & \\
Género & Masculino & 58 & 29.7 \\
Cantidad de enamorados & Femenino & 137 & 70.3 \\
& 1 & 65 & 33.3 \\
Relación sentimental actual & 2 & 52 & 26.7 \\
& Si & 92 & 47.2 \\
Carrera Profesional que estudia & No & 103 & 52.8 \\
& Psicología & 91 & 46.7 \\
& Educación & 27 & 13.8 \\
& Medicina & 21 & 10.8 \\
\hline Religión & Otros & 26 & 13.3 \\
& Católico & 67 & 34.4 \\
& Adventista & 87 & 44.6 \\
& Mormones & 1 & 0.5 \\
& Ninguna & 7 & 3.6 \\
\hline Procedencia & Otros & 31 & 15.9 \\
& Costa & 33 & 16.9 \\
& Sierra & 144 & 73.8 \\
\hline
\end{tabular}

\section{Instrumento}

Cuestionario de Tendencia a la Infidelidad T-IFD por Conde (2009), el cuestionario tiene como objetivo evaluar de manera directa la tendencia que tiene la persona hacia la infidelidad, consta de 33 ítems, la administración es de manera individual y de manera colectiva, el tiempo de aplicación es de 5 a 10 minutos, la confiabilidad por alfa de Cronbach obtenida en el presente estudio es de 0.87 (ver tabla 2) considerado como confiable y aplicable al contexto, por otro lado, la validez por $\mathrm{V}$ de Aiken total es de 0.98 indicando excelente validez (ver tabla 3 ). En cuanto a sus baremos, se clasifica en los siguientes puntajes: Bajo (de 1 a 53), medio (de 76 a 54$)$ y alto (de 77 a 150). 
Escala de Estilos de Amor LAS por Hendrick, C. Y Hendrick. S. (1986), adaptado al español por Rodriguez, Montgomery, Peláez y Salas (2003), el cuestionario tiene como objetivo medir las actitudes hacia el amor, consta de 42 ítems, la administración es de manera individual y de manera colectiva, el tiempo de aplicación es de 5 a 10 minutos. La escala está dividida en 6 subescalas de 7 ítems cada una: Eros, ludus, storge, pragma, manía y ágape. La confiabilidad obtenida por alfa de Cronbach en el presente estudio fue de 0.81 , indicando que el instrumento es confiable y aplicable para el contexto (ver tabla 2), por otra parte, la validez por Vde Aiken total obtenida es de 0.99 indicando excelente validez (ver tabla 3), respecto a su baremación la clasificación de sus puntajes es: Bajo (de 1 a 12), medio (de 13 a 24) y alto (de 25 a 35), considerar que se califica por dimensiones.

Tabla 2

Alfa de Cronbach de los instrumentos utilizados

\begin{tabular}{lll} 
Variables & Alfa de Cronbach & N de elementos/ítems \\
Tendencia a la infidelidad & 0.87 & 33 \\
Estilos de amar & 0.81 & 42 \\
\hline
\end{tabular}

Tabla 3

Validez por $V$ de Aiken de los instrumentos utilizados

\begin{tabular}{|c|c|c|c|c|c|c|}
\hline Instrumentos & Dimensiones & Claridad & Congruencia & $\begin{array}{l}\text { Contex- } \\
\text { to }\end{array}$ & $\begin{array}{l}\text { Dominio del } \\
\text { constructo }\end{array}$ & Total \\
\hline \multirow{5}{*}{$\begin{array}{l}\text { Cuestionario } \\
\text { de Tendencia } \\
\text { a la infidelidad } \\
\text { T-IFD }\end{array}$} & Necesidad de pertenencia & 0.94 & 1.00 & 1.00 & 1.00 & 0.98 \\
\hline & Prejuicios & 0.90 & 1.00 & 0.98 & 1.00 & 0.97 \\
\hline & Diálogo & 0.75 & 1.00 & 1.00 & 1.00 & 0.94 \\
\hline & $\begin{array}{l}\text { Búsqueda de experiencias } \\
\text { nuevas }\end{array}$ & 0.98 & 1.00 & 1.00 & 1.00 & 0.99 \\
\hline & Total & 0.92 & 1.00 & 0.99 & 1.00 & 0.98 \\
\hline \multirow{7}{*}{$\begin{array}{l}\text { Escala de es- } \\
\text { tilos de amor } \\
\text { LAS }\end{array}$} & Eros & 0.96 & 1.00 & 1.00 & 1.00 & 0.99 \\
\hline & Ludus & 1.00 & 1.00 & 1.00 & 1.00 & 1.00 \\
\hline & Storge & 1.00 & 1.00 & 1.00 & 1.00 & 1.00 \\
\hline & Pragma & 1.00 & 1.00 & 1.00 & 1.00 & 1.00 \\
\hline & Manía & 0.86 & 1.00 & 1.00 & 1.00 & 0.96 \\
\hline & Ágape & 1.00 & 1.00 & 1.00 & 1.00 & 1.00 \\
\hline & Total & 0.97 & 1.00 & 1.00 & 1.00 & 0.99 \\
\hline
\end{tabular}

\section{RESULTADOS}

En la tabla 4 se puede observar los niveles obtenidos de cada variable de la población de estudio, tales niveles son distribuidos en tres tipos: Bajo, medio y alto, de la población total el $56.9 \%$ evidencia una tendencia a la infidelidad de nivel medio. En cuanto a los modos de amar, la población que representan un nivel de modos de amar medio son: Eros con un $50.3 \%$, ludus con un $86.7 \%$, manía con un $80.5 \%$ y ágape con un $79.0 \%$, por otro lado, la población que indican un nivel de modos de amar alto son: Storge con un $70.8 \%$ y pragma con un $52.3 \%$.

Las pruebas de normalidad fueron realizadas por el estadístico Kolmogorov-Smirnov(K-S) porque la población era mayor a 50 , los resultados indican que las variables provienen de una distribución no normal, ya que el coeficiente obtenido es $(p<0.05)$, de esta manera los análisis estadísticos correspondientes serán no paramétricas, por lo tanto, se utilizará Tau B de Kendall para las correlaciones (ver tabla 5). 
Tabla 4

Niveles obtenidos de la población por cada variable

\begin{tabular}{|c|c|c|c|c|}
\hline Variables & & Nivel & $\mathrm{n}$ & Porcentaje \\
\hline \multirow{3}{*}{$\begin{array}{l}\text { Cuestionario de Tendencia a la infidelidad } \\
\text { T-IFD }\end{array}$} & \multirow{4}{*}{ Infidelidad } & Bajo & 64 & $32.8 \%$ \\
\hline & & Medio & 111 & $56.9 \%$ \\
\hline & & Alto & 20 & $10.3 \%$ \\
\hline \multirow[t]{9}{*}{ Total } & & & 195 & $100 \%$ \\
\hline & & Bajo & 6 & $3.1 \%$ \\
\hline & Eros & Medio & 98 & $50.3 \%$ \\
\hline & & Alto & 91 & $46.7 \%$ \\
\hline & & Bajo & 18 & $9.2 \%$ \\
\hline & Ludus & Medio & 169 & $86.7 \%$ \\
\hline & & Alto & 8 & $4.1 \%$ \\
\hline & & Bajo & 0 & $0.0 \%$ \\
\hline & Storge & Medio & 57 & $29.2 \%$ \\
\hline \multirow{10}{*}{ Escala de estilos de amor LAS } & & Alto & 138 & $70.8 \%$ \\
\hline & & Bajo & 0 & $0.0 \%$ \\
\hline & Pragma & Medio & 93 & $47.7 \%$ \\
\hline & & Alto & 102 & $52.3 \%$ \\
\hline & & Bajo & 21 & $10.8 \%$ \\
\hline & Manía & Medio & 157 & $80.5 \%$ \\
\hline & & Alto & 17 & $8.7 \%$ \\
\hline & & Bajo & 7 & $3.6 \%$ \\
\hline & Ágape & Medio & 154 & $79.0 \%$ \\
\hline & & Alto & 34 & $17.4 \%$ \\
\hline Total & & & 195 & $100 \%$ \\
\hline
\end{tabular}

Tabla 5

Pruebas de normalidad Kolmogorov-Smirnov

\begin{tabular}{llll} 
& \multicolumn{2}{l}{ Kolmogorov-Smirnov } & \\
\cline { 2 - 4 } Infidelidad & Estadístico & gl & Sig. \\
Eros & .083 & 195 & .002 \\
Ludus & .106 & 195 & .000 \\
Storge & .095 & 195 & .000 \\
Pragma & .137 & 195 & .000 \\
Manía & .071 & 195 & .018 \\
Ágape & .077 & 195 & .006 \\
a. Corrección de significación de Lilliefors & .123 & 195 & .000
\end{tabular}

En la tabla 6 se observa las correlaciones por Tau b de Kendall para las variables, los resultados indican que existe correlación significativa entre tendencia a la infidelidad y los modos de amar ludus $(p=0.00)$, manía $(p=0.00)$, se obtiene una correlación significativa al nivel 0.01 , con un valor $(p<0.05)$. Del mismo modo, se muestra que no existe correlación significativa entre tendencia a la infidelidad y los modos de amar eros $(p=0.338)$, storge $(p=0.178)$, pragma $(p=0.383)$ y ágape $(0.071)$ porque el valor de significancia de estos es $(p>0.05)$. 
Tabla 6

Correlación de las variables tendencia a la infidelidad y estilos de amar. por Tau B de Kendall

\begin{tabular}{|c|c|c|c|c|c|c|c|c|}
\hline & \multirow[b]{2}{*}{ Infidelidad } & \multicolumn{6}{|c|}{ Estilos de amar } \\
\hline & & & Eros & Ludus & Storge & Pragma & Manía & Ágape \\
\hline \multirow{3}{*}{ Infidelidad } & $\begin{array}{l}\text { Coeficiente de } \\
\text { correlación }\end{array}$ & 1.000 & -.048 & $.288^{* *}$ & -.068 & -.044 & $.296^{* *}$ & .091 \\
\hline & Sig. (bilateral) & . & .338 & .000 & .178 & .383 & .000 & .071 \\
\hline & N & 195 & 195 & 195 & 195 & 195 & 195 & 195 \\
\hline
\end{tabular}

\section{DISCUSIÓN}

El estudio tuvo como objetivo determinar si existe relación entre la tendencia a la infidelidad y los modos de amar (eros, ludus, storge, pragma. manía y ágape). A partir del procesamiento estadístico y un nivel de significancia del $5 \%$ los resultados indican que existe correlación entre tendencia a la infidelidad y los modos de amar ludus y manía donde $(p<0.05)$. Sin embargo, no existe correlación significativa con los modos de amar eros, storge, pragma y ágape.

Dichos resultados son similares con otros estudios realizados en Perú, Urizar (2018) encontró relación entre tendencia a la infidelidad y el estilo de amar eros, Delgado (2020) encontró relación estadísticamente significativa entre la infidelidad y el estilo de amar lúdico, obsesivo y erótico, estos sustentan que tales estilos de amar tienen conductas que no implican compromiso en una relación y por esta razón son tendientes a ser más infieles.

Lee (1973) propone 6 estilos de amar, en cuanto al estilo de amar ludus el cual sobresale en correlación a la infidelidad, este refiere que el amor es visto como un juego para el disfrute de ambas parejas o más, no se relaciona con un atractivo físico en especial, ve al sexo como diversión sin implicarse emocionalmente. Tales conductas se estimulan por la era contemporánea, donde el internet y redes sociales amenazan al tipo de amor seguro y altruista (González, 2019). Se vive en la época de las relaciones efímeras, y se puso de moda el no tener compromisos, de esta manera la fidelidad se ve amenazada (Cabanzo, 2017). Lo anterior descrito es similar a la teoría del "amor líquido", este se caracteriza por los aspectos superficiales, hedonistas y el no compromiso de las parejas jóvenes (Vespucci, 2006).

Por otro lado el estilo de amar maníaco, refiere a un tipo de amor posesivo, celoso, intenso en emociones, tiende a la necesidad constante de la otra persona, en cuanto al amor lúdico es razonable entender su tendencia a la infidelidad por su misma forma de amar, en cuanto al modo de amar maníaco, estos son inseguros, celosos, de pensamientos obsesivos, tales conductas son parecidas a los rasgos de personalidad narcisista y paranoide, los cuales tienen alta tendencia a la infidelidad (Mayorga, 2015); asimismo, se encontró que las mujeres tienden a ser infieles cuando se sienten solas e incomprendidas buscando emociones, como amor y atención; en cuanto a los varones, cuando se sienten aburridos, incómodos, confundidos, en algunos casos no sienten culpabilidad o remordimiento por ser infieles, tales características son similares al modo de amor maníaco (Malek, 2015; Valdez et al., 2013). Considerando que las personas con estilo de amar maníaco tienden a desarrollar síntomas de depresión, ansiedad y traumas emocionales (Couch, Baughman y Derow, 2017), también se encontró cambios en la personalidad e inteligencia emocional y en algunos casos síntomas similares al trastorno de estrés postraumático (Verdesoto, Villacis y franco, 2019), de la misma manera se percibe a la infidelidad como una actitud reprochable, causando inseguridades y baja autoestima en la víctima (Bustos, Cáceres y Concha, 2015).

En cuanto al estilo de amar storge no se tiene relación significativa con la infidelidad, esto puede darse por la naturaleza de pensamientos en relación al amor, ya que se caracterizan por ser el amor construido en base a la amistad, 
se busca similitudes con la pareja, confianza, se prioriza el cariño y la amistad más que la apariencia física. El resultado de este estudio respecto a storge es similar al de Álvarez y Garcia (2017) donde las parejas con mayor satisfacción marital tuvieron a storge como principal estilo de amor, se refiere que la relación basada en el compañerismo, la comprensión mutua favorece a la pareja, centrándose en el compromiso y evidenciando cariño; asimismo, para este tipo de personas ser amable con los demás es algo común, mas no una acción de infidelidad.

Resultados diferentes a este estudio respecto al estilo de amar eros y storge fueron encontrados por Espinoza, Correa y García (2014), en su estudio realizado "Percepción social de la infidelidad y estilos de amar en la pareja" se analizó la percepción de infidelidad masculina, se halló una correlación positiva en el estilo eros y storge, donde ambos imparten conceptos distintos, ya que los eros se centran más en el físico y satisfacción sexual, debido a esto deducen que su pareja tiene el mismo interés; en síntesis ser infiel para éste tipo de personas es algo habitual. Posiblemente los resultados sean diferentes por la diferencia de culturas, considerando que los ítems en la dimensión eros de este estudio están enfocados en una sola pareja.

Marzee y Lukasik (2017), encontraron resultados similares al presente estudio, donde personas con estilo de amar eros, storge y pragma tenían tendencia a relaciones a largo plazo, por otro lado, ludus se le vincula con relaciones cortas. Resultados del estudio anterior y demás investigaciones, en contraste con el presente estudio confirman que ludus está normalmente relacionada con la infidelidad, sin embargo, se difiere en los demás estilos de amor como eros, ya que en otros estudios si encuentran relación con la infidelidad y en otros no.

De la misma manera, este estudio evidencia que los modos de amar ágape y pragma son los que no tienen relación hacia la infidelidad, esto se explica porque el estilo de amar altruista se basa en un amor honesto y comprensivo, brindando apoyo y amor sin ningún tipo de interés de por medio, perdonando sin dificultad. En cuanto al modo de amar pragmático, éste busca más compatibilidad de condición (ya sea de carácter o costumbre), seleccionando el nivel social y educativo, pretendiendo saber todo respecto a su pareja previo a tener intimidad, viendo su conveniencia hacia un futuro (Delgado, 2020; Büyükflachim y Hovardaolu, 2004).

Teniendo en cuenta que existen muchos factores relacionados a la infidelidad, como el machismo (Puicon y Salas, 2016), tiempo de convivencia, edad (Quevedo, 1997), género (Malek, 2015), violencia física y/o psicológica (Romero, Romero y Arellano, 2017), internet y redes sociales (Cruz y Rodríguez, 2016; Gonzalez, 2019), etc; el modo de amar que tenga una persona no determina si será infiel o no, esto según los resultados encontrados en el presente estudio y contrastados con otros similares; sin embargo, las conductas de los factores antes mencionados son iguales a las características de los diferentes modos de amar, de esta manera algunos modos de amar tienen más tendencia a la infidelidad que otros.

\section{Declaración de financiamiento y de conflictos de interés:}

El estudio fue financiado por los autores, quienes declaran no tener algún tipo de conflicto de interés en la investigación realizada.

\section{Correspondencia}

\section{Fernando Pari Belizario}

Correo electrónico:

fernandoparbel@gmail.com

\section{REFERENCIAS}

Alavi, M., Kye Mei, T., \& Mehrinezhad, S. A. (2018). The Dark Triad of personality and infidelity intentions: The moderating role of relationship experience. Personality and Individual Differences, 128, 4954. https://doi.org/10.1016/j.paid.2018.02.023

Alva Díaz, M. D. P. (2017). Elaboración de una escala de infidelidad en adultos del distrito de Santiago de Surco, Lima - Perú, 2017. Universidad César Vallejo. Universidad César Vallejo, Lima. http://repositorio.ucv.edu.pe/ handle/20.500.12692/11375

Álvarez Ramírez, E., \& García Méndez, M. (2017). Estilos de amor y culpa como predictores de la satisfacción marital en hombres y mujeres. Enseñanza e Investigación en Psicología, 22(1), 76-85. Recuperado de https://www.redalyc.org/ articulo. oa? id=29251161007 
Bustos Riquelme, F. M., Cáceres Urra, M. P., \& Concha Salas, C. A. (2015). Percepción de la infidelidad y su influencia en el. Universidad de Ciencias de la Informática. Recuperado de http:// repositorio.ugm.cl/handle/20.500.12743/527

Büyükflachim, A., \& Hovardaolu, A. (2004). Un estudio de las actitudes hacia el amor en parejas. Revista de Psicología.

Cabanzo de Ponce de León, R. (2017). Infidelidades en la era contemporanea. Vinculo, 14(1), 1-8. Recuperado de http://pepsic.bvsalud.org/ scielo.php?script $=$ sci_abstract\&pid=S1806$24902017000100002 \&$ Ing=pt\&nrm=iso\&tIng=es

Cassepp-Borges, V., \& Pasquali, L. (2012). Estudo nacional dos atributos psicométricos da escala Triangular do Amor de Sternberg. Paideia, 22(51), 21-31. https://doi.org/10.1590/S0103863X2012000100004

Chávez Sopla, E. K., \& Juárez Paz, K. Y. (2016). Relación entre la satisfacción sexual y la infidelidad en pacientes de 20 - 40 años atendidos en el hospital distrital de Laredo durante el año 2013. Universidad Privada Antenor Orrego. Universidad Privada Antenor Orrego - UPAO, Trujillo. http:// repositorio.upao.edu.pe/handle/upaorep/1703

Conde, M. (2009). Estilos de comunicación y Tendencia a la infidelidad en estudiantes de una universidad privada.

Couch, L. L., Baughman, K. R., \& Derow, M. R. (2017). The Aftermath of Romantic Betrayal: What's Love Got to Do with It? Current Psychology, 36(3), 504515. https://doi.org/10.1007/s12144-016-9438-y

Crus Fernandez, F. F., \& Rodriguez Contreras, R. C. (2016). Uso de redes sociales y actitudes frente a la infidelidad en estudiantes universitarios. Universidad Católica De Santa María, Arequipa. https://alicia.concytec.gob.pe/vufind/Record/ UCSM_e56715f383b46c7c86276789cd63e806

De Roda, A. B. L., Martínez-Íñigo, D., de Paúl, P., \& Yela, C. (1999). Romantic Beliefs and Myths in Spain. The Spanish Journal of Psychology, 2(1), 64-73. https://doi.org/10.1017/S1138741600005461

DelgadoNieto,D.M.(2020). Estilos deamareinfidelidad en estudiantes universitarios. Universidad Católica de Santa María, Arequipa. Recuperado de https://alicia.concytec.gob.pe/vufind/Record/ UCSM_74857f6c32349ac6688cd73e0a366c11

El Comercio (2010). La mayoría de latinoamericanos son infieles, concluyó encuesta sobre sexualidad. https://archivo.elcomercio.pe/mundo/actualidad/ mayoria-latinoamericanos-son-infieles-concluyoencuesta-sobre-sexualidad-noticia-658674
Espinoza Romo, A. V., Correa Romero, F. E., \& García y Barragán, L. F. (2014). Percepción social de la infidelidad y estilos de amor en la pareja. Enseñanza e Investigación en Psicología, 19(1), 135-147. http://www.redalyc.org/articulo. oa?id=29232614008

Fisher, H. (2004). Por qué amamos. Naturaleza y química del amor romántico. (S. E. Generales, Ed.). México.

Garciandía Imaz, A. J., \& Samper Alum, J. (2012). Las infidelidades: aprendiendo desde dentro de las conversaciones terapéuticas. Rev. Colomb. Psiquiat, 41(3), 496-520. Recuperado de http:// www.scielo.org.co/pdf/rcp/v41n3/v41n3a04.pdf

González Rivera, J. A. (2019). Conductas relacionadas a la infidelidad en las Redes Sociales: validación y estudio psicométrico. Informes psicológicos, 19(2), 43-51. https://doi.org/10.18566/infpsic. v19n2a03

Hendrick, C., \& Hendrick, S. (1986). A Theory and Method of Love. Journal of Personality and Social Psychology, 50(2), 392-402. https://doi. org/10.1037/0022-3514.50.2.392

Hernández, R., Fernández, C., \& Baptista, P. (2014). Metodología de la Investigación (Sexta edic). México: Mc Graw Hill.

Lee, J. (1973). Los colores del amor: una exploración de las formas de amar. (N. Prensa, Ed.). Toronto.

Lewandowski, G. W., \& Ackerman, R. A. (2006). Something's missing: Need fulfillment and self-expansion as predictors of susceptibility to infidelity. Journal of Social Psychology, 146(4), 389-403. https://doi.org/10.3200/ SOCP.146.4.389-403

Maldonado Cañola, E. M. (2018). Tendencia Hacia la Infidelidad y Actitudes Hacia el Machismo en Estudiantes Varones de la Facultad de Ingeniería de Minas de una Universidad De Piura- 2018. Universidad César Vallejo. Universidad César Vallejo, Piura. https://repositorio.ucv.edu.pe/ handle/20.500.12692/29131

Malek, N. (2015). La monogamia y la justificación de la infidelidad, diferencias de género. Tesis de licenciatura. Universidad Del Aconcagua.

Marzec, M., \& Łukasik, A. (2017). Love Styles in the Context of Life History Theory. Polish Psychological Bulletin, 48(2), 237-249. https:// doi.org/10.1515/ppb-2017-0027

Mayorga Zapata, D. S. (2015). Rasgos de personalidad y su influencia en la infidelidad de las personas de 18 a 50 años de edad que acuden al centro de terapia psicológica integral de la ciudad de Ambato. Universidad 
Técnica de Ambato, Ambato. http://repositorio. uta.edu.ec/bitstream/123456789/10338/1/ MayorgaZapata\%2CDavidSantiago.pdf

Pedrós Mestre, A. (2016). Infidelidad, estilos de amor y micromachismos. Universitat Jaume, Castellón. https://core.ac.uk/download/pdf/61487116.pdf

Puicón Túllume, C. T., \& Salas Soriano, J. E. (2016). Satisfacción sexual y predisposición hacia la infidelidad en mujeres de Chiclayo, 2016. Repositorio Institucional - USS. Universidad Señor de Sipán, Chiclayo. http://repositorio.uss. edu.pe/xmlui/handle/uss/4127

Quevedo Pereyra, R. J. (1997). Archivos Hispanoamericanos de sexología. Archivos hispanoamericanos de sexología (Vol. 3). https://biblat.unam.mx/es/revista/archivoshispanoamericanos-de-sexologia/articulo/ actitudes-hacia-la-infidelidad-en-miembros-deparejas-conyugales-en-lima-metropolitana

Ramirez Febre, P. E. (2017). Relación entre el clima social familiar y la tendencia a la infidelidad en el alumnado del IV, VII y VIII ciclo de la escuela profesional de psicología de la Universidad Católica los Ángeles de Chimbote. Piura, 2015. Repositorio Institucional - ULADECH. Universidad Católica Los Ángeles de Chimbote, Piura. http://repositorio.uladech.edu.pe/ handle/123456789/129

Rodríguez, I., Montgomery, M., Peláez, M., y Salas, W. (2003). Actitudes amorosas y experiencias en el cortejo de adultos y jóvenes de tres distintas culturas. Revista Mexicana de Psicología, 20 (2), 177-188. https://faculty.fiu.edu/ pelaeznm/wpcontent/uploads/2016/10/14.Love-attitudes-anddating-experiences.pdf

REAL ACADEMIA ESPAÑOLA. (s. f.). Modo de cita | Diccionario de la lengua española | Edición del Tricentenario (23.a ed.). https://dle.rae.es/ contenido/cita

Romero, H., Romero, L., \& Arellano, J. (2017). La infidelidad femenina como producto de la violencia intrafamiliar Female infidelity as a result of domestic violence. An Fac med, 78(2), 161165. https://doi.org/10.15381/ana

Romero Palencia, A., RiveraAragón, S., \& Loving, R. D. (2007). Desarrollo del inventario multidimensional de infidelidad (IMIN). Revista Iberoamericana de Diagnóstico y Evaluación, 1(23), 121-147. http:// www.redalyc.org/articulo.oa? $\mathrm{id}=459645446008$

Urizar Ocharan, A. J. (2018). Tendencia a la Infidelidad y Estilos de Amar en Estudiantes de Posgrado de una Universidad Pública de Arequipa. Universidad Católica de Santa María, Arequipa. https://alicia.concytec.gob.pe/vufind/Record/ UCSM_71425661f349d85ad5db0a29bb65e398
Urrego Betancourt, Y., Gaitán Rodríguez, N. A., \& Umbarila Forero, D. A. (2016). Relación entre el tipo de apego y la conducta de infidelidad en adultos jóvenes. Revista de Psicología, 12(24), 41-54. https://repositorio.uca.edu.ar/ handle/123456789/6146

Valdez Medina, J. L., González Colín, B., Maya Martínez, M. U., Aguilar Montes de Oca, Y. P., González Arratia López Fuentes, N. I., \& Torres Muñoz, M. A. (2013). Las causas que llevan a la infidelidad: Un análisis por sexo. Acta de investigación psicológica, 3(3), 1271-1279. http://www.scielo.org.mx/scielo.php?script=sci_ arttext\&pid=S2007-48322013000300007

Vargas, J., \& Ibañez, J. (2008). Solucionando los problemas de pareja y familia. (P. México, Ed.) (Cuarta edi). México.

Vargas Londoño, A. D. P. (2011). "Poder y placer: ¿cuál es el impacto que la interseccionalidad entre género y clase social tiene sobre las prácticas sexuales? Un estudio comparativo en torno a la infidelidad, sexo casual, uso de lencería, ataduras e inmovilizaciones, piropos, sexo oral, orgías y encuentros con prostitutas en hombres y mujeres en los sectores populares y medio-altos en Bogotá, Colombia.".

Verdesoto Galeas, J. del R., Villacís Verdesoto, M. V., \& Franco CHoez, X. E. (2019). Impacto emocional de la infidelidad en las relaciones de pareja. Importancia de su conocimiento para el psicólogo clínico. Opuntia Brava, 349-361. http:// opuntiabrava.ult.edu.cu/index.php/opuntiabrava/ article/view/883

Vespucci, G. (2006). Amor líquido. acerca de la fragilidad de los vínculos humanos. Revista Argentina de Sociología, 4(6), 160-163. http:// www.redalyc. org/articulo.oa? $i d=26940608$

Weiser, D. A., Niehuis, S., Flora, J., Punyanunt-Carter, N. M., Arias, V. S., \& Hannah Baird, R. (2018). Swiping right: Sociosexuality, intentions to engage in infidelity, and infidelity experiences on Tinder. Personality and Individual Differences, 133, 2933. https://doi.org/10.1016/j.paid.2017.10.025

Yela, C. (2001). El Amor desde la Psicología Social: ni tan libres, ni tan racionales. Psicothema, 13(2), 335-336. https://www.redalyc.org/articulo. oa?id=72721325

Recibido: 11/06/2021 Aceptado: 12/09/2021 